\title{
Substrate polarization effect on the band gaps of one- dimensional semiconducting atomic wires
}

\author{
Adam J. Simbeck ${ }^{1,5}$, Nicholas A. Lanzillo ${ }^{1}$, Neerav Kharche ${ }^{1,2,3}$, and Saroj K. Nayak ${ }^{1,4}$ \\ ${ }^{1}$ Department of Physics, Applied Physics, and Astronomy, Rensselaer Polytechnic \\ Institute, Troy, NY, 12180, USA \\ ${ }^{2}$ Computational Center for Nanotechnology Innovations, Rensselaer Polytechnic Institute, \\ Troy, NY 12180, USA \\ ${ }^{3}$ Chemistry Department, Brookhaven National Laboratory, Upton, NY 11973, USA \\ ${ }^{4}$ Indian Institute of Technology Bhubaneswar, 751013, Bhubaneswar, India \\ E-mail: adamjsimbeck@gmail.com, lanzin2@ rpi.edu, nkharche@bnl.gov, \\ nayaks@iitbbs.ac.in
}

\begin{abstract}
The dielectric screening induced modulation of the electronic structure of model $\mathrm{SiH}_{2}$ and $\mathrm{GeH}_{2}$ onedimensional atomic wires is investigated using graphene as a prototypical substrate. A combination of firstprinciples density functional theory and many-body perturbation theory within the GW approximation is employed to investigate how the substrate alters the electronic structure of the weakly bounds wires. The quasiparticle GW band gaps of the atomic wires are reduced by $\sim 1 \mathrm{eV}$ when supported by a graphene substrate. The band gap reduction is attributed to a change in the correlation energy of the frontier orbitals of the atomic wires due to the increased effective screening of the Coulomb interaction as a result of the polarization of the dielectric substrate. This work indicates that the band gaps of semiconducting nanowires composed of $\mathrm{Si}$ and $\mathrm{Ge}$ can be engineered via the interaction with the substrate in addition to conventional approaches such as adjusting size and crystal orientation.
\end{abstract}

Submitted to: Journal of Physics Condensed Matter

\footnotetext{
${ }^{5}$ Author to whom correspondence should be addressed: adamjsimbeck@gmail.com
} 
1. Introduction. While a number of potential candidates for the miniaturization of active integrated circuit components have been explored over the last two decades, none have shown more promise than $\mathrm{Si}$ and Ge semiconducting nanowires. ${ }^{1}$ Not only do such wires present a number of options for tuning their electronic properties, such as size, orientation, and composition, ${ }^{2-6}$ but the ability to experimentally fabricate small diameter (down to $\sim 1 \mathrm{~nm}$ ) wires and implement them in prototypical devices has already been achieved. ${ }^{7-12}$ It has even been experimentally demonstrated that such nanowires can be used as the basic building blocks in more complicated circuit components. ${ }^{711}$ Similar potential surrounds carbonbased nanoelectronics, ${ }^{13}$ but carbon-based devices lack the ease of integration and natural compatibility of Si and Ge with current semiconductor technology.

Whereas the effect of geometry, size, orientation, composition, and surface passivation on the electronic structure of $\mathrm{Si}$ and Ge nanowires has been exhaustively studied, ${ }^{2-4,14-21}$ the role played by the dielectric environment surrounding the wire, in particular the substrate upon which the wire is supported, has seen considerably less attention. ${ }^{21-24}$ This is thought to be rather important though since both experiments and devices involving such wires will require supporting them on a substrate. Using a combination of tight-binding (TB) and a semi-analytical self-energy correction model, Niquet et al. found that a dielectric mismatch between the nanowires and their environment can substantially open up the band gap when the dielectric constant of the wire exceeds that of its environment. ${ }^{21}$ On the other hand, when the dielectric constant of the environment was allowed to exceed that of the wire the self-energy corrections were reduced compared to the previous case. Although this trend is consistent with the understanding of other reports for the effect of the dielectric environment on the band gap, ${ }^{25-30}$ the results of Ref. 21 were found to be of limited quantitative accuracy in the regime where the dielectric constant of the wire is less than that of the environment. Rather, a more complete quantitative description of such trends requires the use of many-body perturbation theory (MBPT).

In this work, ultrathin, H-passivated, and [110]-oriented one-dimensional (1D) atomic wires (AWs) composed of $\mathrm{Si}$ and Ge are studied with and without a graphene substrate (dielectric) from first[Type text] 
principles. The small model atomic wires are chosen to reduce computational cost in the MBPT calculations. Graphene is chosen as a substrate for the following reasons: lack of surface dangling bonds, weak interaction with the fully passivated wire, and minimal number of unit cell atoms which again makes the MBPT calculations more tractable. The weak interaction between the wire and graphene is required such that the electronic structure modulation can be ascribed to the dielectric screening, and not any strong, chemical interaction. In any case, since our goal is to focus on this effect when the wiresubstrate interaction is weak, graphene provides a good model. The band gaps in the free-standing configuration are found to be in good agreement with the previously reported trends for $\mathrm{Si}$ and $\mathrm{Ge}$ nanowires. ${ }^{2-4,15-20}$ For the graphene-supported AWs, it is found that the substrate-induced polarization renormalizes the AW energy levels such that the band gap is suppressed by $\sim 1.1 \mathrm{eV}$, in agreement with the previously reported trend..$^{21}$ The gap renormalization is ascribed to a reduction in the screened Coulomb interaction and is compared to other systems where substrate-induced band gap suppression has been reported. ${ }^{25-30}$

2. Methods. The density functional theory (DFT) $)^{31,32}$ and MBPT calculations were performed using the ABINIT code. ${ }^{33,34}$ The electron-ion interaction is described using norm-conserving pseudopotentials generated using the Trouiller-Martins scheme. ${ }^{35}$ The local density approximation (LDA) of Teter-Pade ${ }^{36}$ is employed to describe the exchange-correlation energy. Convergence testing for the DFT results for the free-standing wires revealed that an energy cutoff of $38.0 \mathrm{Ha}$, a $1 \times 1 \times 30 \mathrm{k}$-point sampling, and at least 10 $\AA$ of vacuum separating periodic images is necessary to pin down the total energy to within $0.5 \mathrm{mHa}$. Identical parameters were employed for the DFT calculations where the wires are supported by a graphene substrate.

Here it is pointed out that rather than strain the AW to match the lattice of the substrate, the substrate has been strained such that the AW can simply be placed atop it. At its maximum, this compressive strain is approximately $11 \%$ and does not drastically alter the electronic structure of the substrate. For example, the Dirac cone present at the K-point in the band structure of graphene, and hence the semi-metallic [Type text] 


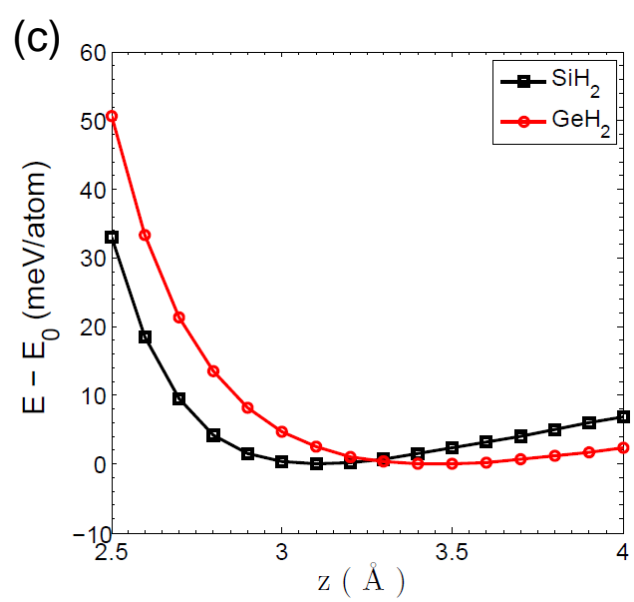

Figure 1. Geometry of the $\mathrm{SiH}_{2}$ and $\mathrm{GeH}_{2} \mathrm{AWs}$ (a) in the free-standing configuration as viewed along the length of the wire and (b) in the substrate-supported configuration as viewed along the cross-section. The $\mathrm{Si} / \mathrm{Ge}$ atoms appear as large, blue (dark) circles and the $\mathrm{H}$ atoms as small, gray ones. Total relative energy as a function of substrate separation $(\mathrm{z})$ is shown in $(\mathrm{c})$

nature of the substrate, is preserved [see figures 2(b), (d)], consistent with the results of Ref. 37. A similar methodology has been adopted in Ref. 38 .

The composite system is generated by first straining the substrate cell to match the periodicity of the AW. It is reiterated here that even at a compressive strain of $11 \%$, the fundamental electronic properties of the graphene substrate are preserved, i.e., no gap is opened up at the Dirac cone and graphene remains semi-metallic. A rectangular supercell is then built from the strained, hexagonal unit cell and the AW is combined with the substrate at a separation of $z=3.25 \AA$. In total, each composite system contains 42 atoms: 36 substrate $(\mathrm{C})$ atoms and $6 \mathrm{AW}(2 \mathrm{Si} / \mathrm{Ge}, 4 \mathrm{H})$ atoms. For the remainder of this work the composite systems which feature a graphene $(\mathrm{G})$ substrate shall be referred to as $\mathrm{SiH}_{2} / \mathrm{G}$ and $\mathrm{GeH}_{2} / \mathrm{G}$.

The geometry of the [110] free-standing AWs [figure 1(a) $]^{39}$ and bare graphene substrate was allowed to fully relax (with the lattice constant fixed) until the maximum force was less than $1 \times 10^{-5} \mathrm{Ha} / \mathrm{Bohr}$. The wire was then combined with a rectangular graphene supercell by placing it at a separation of $z=3.25 \AA$ from the substrate [figure 1(b)]. The wire-substrate separation $z$ was chosen to reflect the weak, van der Waals interaction that is expected to exist between the dangling-bond-free graphene surface and the hydrogenated AWs. Figure 1(c) shows the total relative energy as a function of $\mathrm{z}$ and as expected, shallow 
minima are observed in the range of $3.1 \AA$ for $\mathrm{SiH}_{2}$ and $3.4 \AA$ for $\mathrm{GeH}_{2}$, which is characteristic of a weak van der Waals interaction. Any further lattice mismatch or rotations with respect to the substrate have not been considered and are outside the scope of this work, although based on the results of reference 27 , small changes in the wire/substrate orientation are not expected to dramatically influence the electronic structure. The length of the supercell $b$ was chosen so that the separation between wires along the substrate surface was almost $18 \AA$. The supercell length also ensures that the Dirac cone associated with the K-point of the hexagonal unit cell of graphene is mapped to the $\Gamma$-point of the rectangular supercell [figures 2(b), (d)].

The tolerance for the energy calculations was set at $1 \times 10^{-6} \mathrm{Ha}$ and the band structure calculations utilized a wavefunction squared residual tolerance of $1 \times 10^{-12} \mathrm{Ha}^{2}$. This combination of convergence and cutoff parameters allowed the DFT predicted gaps for the free-standing and graphene supported AWs to be converged to within $10 \mathrm{meV}$ or better. Note that the motivation behind examining a composite of an ultrathin [110] wire and a one atom thick substrate is to capture the role of the substrate in altering the electronic structure of the weakly bound wire while maintaining a model which is both computationally tractable and efficient. Similar simple models have been shown to provide converged results while also lending insight into the underlying physics. ${ }^{30}$

Since it is well known that DFT drastically underestimates the band gap, ${ }^{40,41}$ quasiparticle corrections to the DFT electronic structure employing MBPT within the GW $(G=$ Green's function, $W=$ screened Coulomb potential) approximation ${ }^{42}$ have been carried out. For all GW calculations the Coulomb cutoff scheme proposed by Ismail-Beigi ${ }^{43}$ is employed and screening is calculated using the Hybertsen-Louie plasmon pole model. ${ }^{44}$ To converge the quasiparticle gaps to within $\sim 0.2 \mathrm{eV}$, it is necessary to employ a much more aggressive k-point sampling. A $1 \times 1 \times 78 \mathrm{k}$-point grid was used for the free-standing wires and a $30 \times 4 \times 1$ grid for the wires on graphene. A similar, dense k-point sampling was adopted in Ref. 20 and 45. A double-check of the convergence of the GW gaps with respect to the vacuum was also performed and it was found that increasing the vacuum by $5 \AA$ only changes the GW gaps by $\sim 30 \mathrm{meV}$ or less for 
Table 1. Summary of geometry for free-standing $\mathrm{SiH}_{2}$ and $\mathrm{GeH}_{2} \mathrm{AWs}$. Refer to figure 1 for the labeling conventions.

\begin{tabular}{ccccc}
\hline $\mathrm{AW}$ & $\mathrm{c}(\AA)$ & $\mathrm{d}(\AA)$ & $\mathrm{h}(\AA)$ & $\alpha\left(^{\circ}\right)$ \\
\hline $\mathrm{SiH}_{2}$ & 3.84 & 2.32 & 1.49 & 111.9 \\
$\mathrm{GeH}_{2}$ & 3.92 & 2.36 & 1.53 & 112.4 \\
\hline
\end{tabular}

both the free-standing and substrate supported AWs. All the GW simulations employed an energy cutoff of $3 \mathrm{Ha}$ and 300 bands in the dielectric and self-energy calculations.

3. Results. The geometry of the free-standing and substrate supported systems are featured in figure 1 and table 1. The free-standing [110] AWs are composed of either Si or Ge and each atom of the wire has been passivated with two $\mathrm{H}$ atoms. The [110] direction has been selected since it has been found to be a favorable growth orientation in experiment. ${ }^{46,47}$ Both the $\mathrm{SiH}_{2}$ and the $\mathrm{GeH}_{2}$ wires adopt a wide zigzag geometry [figure 1(a)] with almost identical bonding (table 1). The geometry for the $\mathrm{SiH}_{2}$ wire is in good agreement with the theoretical results of Ref. 14 and 48, as well as the experimental bonding parameters reported in Ref. 2 for Si nanowires of larger cross-sectional area.

The results for the electronic structure of both the free-standing and graphene supported AWs are featured in figure 2 and table 2. Before analyzing the electronic structure, it is pointed out that a number of characteristics can be ascertained from the extensive literature on $\mathrm{Si}$ and Ge nanowires. For example, both [110] AWs are expected to be direct band gap semiconductors because of their small size and the fact that the bulk conduction band minimum will be mapped to the $\Gamma$-point for the [110] wire orientation. ${ }^{4,15,16,18}$ The DFT/GW gaps and GW corrections of the AWs are also expected to be much larger than that of their bulk, or larger cross-sectional area nanowire, counterparts due to quantum confinement. ${ }^{2-4,15-20}$ Furthermore, the uppermost valence band and lowest conduction band should show a relatively large dispersion due to the delocalized nature of these states along the axis of the wire. ${ }^{16,18}$

From figures 2(a) and 2(c) both AWs are indeed direct band gap semiconductors with their fundamental gaps located at $\Gamma$. In addition, the highest valence band and lowest conduction band are largely dispersive, indicating a low hole and electron effective mass, respectively. The DFT gaps for the free-standing wires are $3.34 \mathrm{eV}$ and $3.00 \mathrm{eV}$ for the $\mathrm{SiH}_{2}$ and $\mathrm{GeH}_{2} \mathrm{AWs}$, respectively. The electronic [Type text] 
structure of the $\mathrm{SiH}_{2}$ wire is in good agreement with Ref. 14 and 48. Furthermore, the DFT gaps for both AWs are found to be larger than those reported for similarly oriented Si and Ge nanowires with larger cross-sections, in agreement with the inverse relationship between the gap and the wire diameter. ${ }^{4,15,17}$ Lastly, the $\mathrm{SiH}_{2} \mathrm{AW}$ gap is larger than the $\mathrm{GeH}_{2}$ AW gap, in accordance with the trends of Ref. 15, 17, and 20 , as well as the bulk. 
(a) $\mathrm{SiH}_{2}$

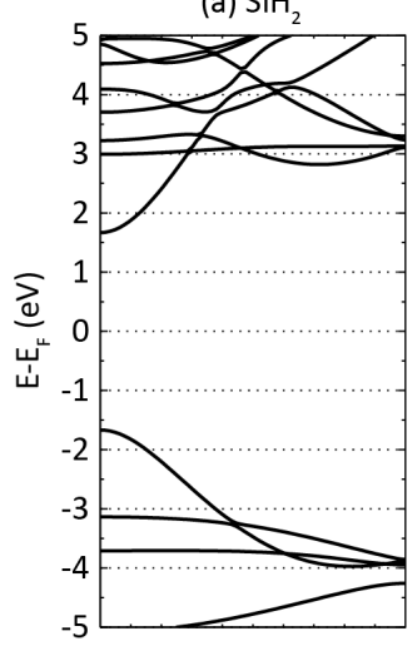

(c) $\mathrm{GeH}_{2}$

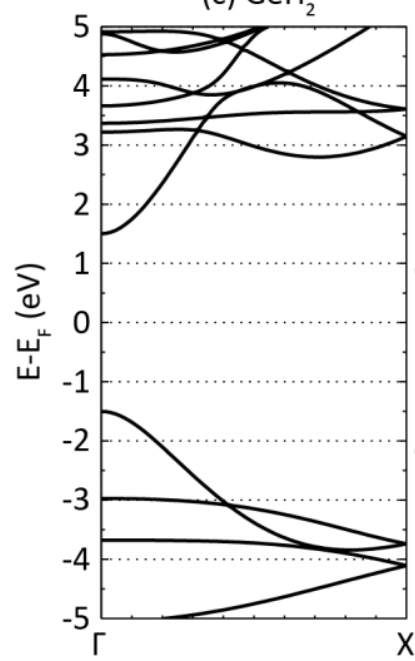

(b) $\mathrm{SiH}_{2} / \mathrm{G}$

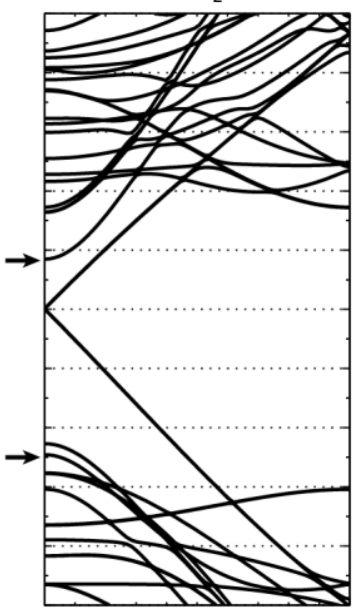

(d) $\mathrm{GeH}_{2} / \mathrm{G}$

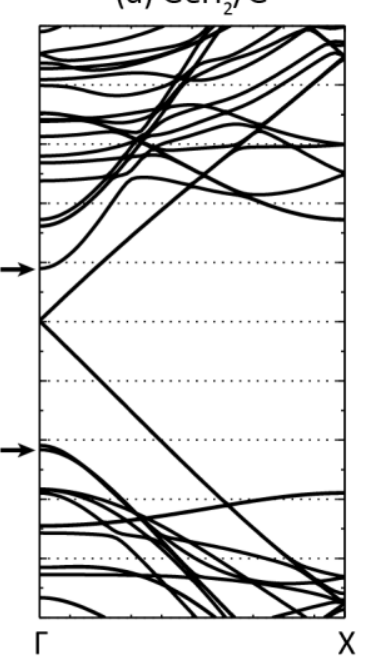

Figure. 2. DFT band structure of (a) $\mathrm{SiH}_{2}$, (b) $\mathrm{SiH}_{2} / \mathrm{G}$, (c) $\mathrm{GeH}_{2}$, and (d) $\mathrm{GeH}_{2} / \mathrm{G}$. For the composite systems, the valence band maxima and conduction band minima of the AWs at the $\Gamma$-point have been highlighted by arrows. The bands of the free-standing AWs are centered on the gap.

Table 2 highlights the results for the GW calculations for the free-standing systems. For both wires the gap is predicted to increase: from $3.34 \mathrm{eV}$ (DFT) to $6.62 \mathrm{eV}(\mathrm{GW})$ for $\mathrm{SiH}_{2}$ and from $3.00 \mathrm{eV}$ (DFT) to $5.93 \mathrm{eV}(\mathrm{GW})$ for $\mathrm{GeH}_{2}$. Lu et al. also considered MBPT corrections to the DFT-LDA gap of the $\mathrm{SiH}_{2}$ $\mathrm{AW},{ }^{48}$ but the enhancement reported here is much stronger. This discrepancy is most likely due to under convergence with respect to vacuum spacing and k-point sampling, as well as the lack of employing a Coulomb cutoff scheme to truncate long-range interactions between periodic images in Ref. 48. The efforts of Peelaers et al. have shown such parameters to be crucial in establishing convergence of the [Type text] 
Table 2. Summary of the quasiparticle band gaps for free-standing and graphene-supported $\mathrm{SiH}_{2}$ and $\mathrm{GeH}_{2} \mathrm{AWs}$. The change in the gap $\Delta$ is the reduction due to the substrate, i.e., the free-standing (AW) gap less the substrate-supported $(\mathrm{AW} / \mathrm{G})$ gap.

\begin{tabular}{cccc}
\hline & $\begin{array}{c}\text { AW } \\
\text { GW Gap } \\
(\mathrm{eV})\end{array}$ & $\begin{array}{c}\text { AW/G } \\
\text { GW Gap } \\
(\mathrm{eV})\end{array}$ & $\begin{array}{c}\Delta \\
(\mathrm{eV})\end{array}$ \\
\hline $\mathrm{SiH}_{2}$ & 6.62 & 5.47 & 1.15 \\
$\mathrm{GeH}_{2}$ & 5.93 & 4.85 & 1.08 \\
\hline
\end{tabular}

quasiparticle band structure of Si and Ge nanowires. ${ }^{20}$ The GW corrections for the ultrathin [110]-oriented semiconducting atomic wires are also found to agree with the trends reported for similarly oriented, larger diameter Si and Ge nanowires in Ref. 4, 15, 17, 18, and 20, i.e., the corrections for the AWs are larger because of increased quantum confinement. It is interesting to note that, for the $\mathrm{SiH}_{2} \mathrm{AW}$, the GW correction is slightly larger than that of the $\mathrm{GeH}_{2} \mathrm{AW}$, a trend which appears to be independent of wire size and orientation. ${ }^{15,17,20}$ Lastly, as a function of the AW-G separation $z$, it is anticipated that as the AW moves closer to (further from) the substrate, the band gap reduction will be enhanced (suppressed), similar to the results of Ref. 30 for semiconducting carbon nanotubes (CNTs) on hexagonal boron nitride $(\mathrm{hBN})$.

Figures 2(b) and 2(d) show that the electronic structure of the AWs from DFT is relatively unchanged with the addition of the graphene substrate. For both wires the valence band maximum and conduction band minimum, which have been highlighted at the $\Gamma$-point by black arrows, are still clearly visible. In addition, the characteristic Dirac cone of graphene is mapped to the $\Gamma$-point, which for the composite lies in the band gap region of the AWs but closer to the conduction band minima of the AWs. Note that the change in the DFT predicted gap in the presence of the substrate is negligible within the accuracy of our method. The quasiparticle calculations on the other hand show that the substrate plays a critical role in modifying the band gaps of the AWs. For the case of $\mathrm{SiH}_{2} / \mathrm{G}$, the AW gap is reduced by $1.15 \mathrm{eV}$, and for $\mathrm{GeH}_{2} / \mathrm{G}$ the reduction is $1.08 \mathrm{eV}$ (table 2).

4. Discussion. The mechanism behind the band gap suppression is identical to that reported in previous works for molecules on metals, ${ }^{25,26}$ graphone on $\mathrm{hBN},{ }^{27}$ graphene nanoribbons on $\mathrm{Au}^{28}$ and $\mathrm{hBN},{ }^{29}$ and $\mathrm{CNTs}$ on $\mathrm{hBN} .{ }^{30}$ In short, the self-energy of the electrons is modified by the presence of a [Type text] 
dielectric (graphene substrate). More specifically, the polarization of the substrate reduces the screened Coulomb interaction $(W)$ which in turn suppresses the band gap of the AW compared to its free-standing counterpart. This non-local, long-range screening effect is completely missed by any local or semi-local approximation to the exchange-correlation energy in DFT because of a failure to reproduce the asymptotic nature of the image potential tail. ${ }^{49,50}$ In closing this section, it is also noted that the GW gap reduction reported here is smaller than previously reported values for molecules on metals $(\sim 2-3 \mathrm{eV}),{ }^{25,26}$ but larger than most carbon nanostructures supported by various metallic and semiconducting substrates $(\sim 0.4-0.8 \mathrm{eV}){ }^{27-30}$ This trend is attributed to the one-dimensional nature of the AWs considered, which compared to the previously studied molecules (carbon nanostructures) are less (more) confined. Recent work also suggests that including more than one graphene layer as a substrate is not likely to influence the quasiparticle band gaps ${ }^{51}$.

5. Conclusion. In summary, a combination of first-principles density functional theory and manybody perturbation theory has been employed to analyze the electronic structure of ultrathin, H-passivated [110] Si and Ge wires with and without a graphene substrate. Whereas the density functional calculations show no modulation of the fundamental gaps of the semiconducting AWs upon the introduction of the substrate, the GW calculations find a large reduction of the band gaps: $1.15 \mathrm{eV}$ for $\mathrm{SiH}_{2}$ and $1.08 \mathrm{eV}$ for $\mathrm{GeH}_{2}$. This reduction is attributed to a substrate-induced polarization effect, which in-turn more effectively screens the Coulomb potential and hence reduces the band gap of the semiconducting AWs. These results extend the work on substrate (dielectric) screening to wires composed of typical semiconductors, i.e., $\mathrm{Si}$ and $\mathrm{Ge}$, and are expected to be useful as experimental progress is made in controlling the growth, diameter, and orientation of Si and Ge wires. Furthermore, the results show that besides manipulating the size, orientation, and composition of the wire, the choice of the substrate lends yet another avenue for engineering the band gap. For future work, it will be interesting to investigate the substrate-induced band gap reduction as a function of wire cross-section, orientation, composition, and doping, as well as substrate composition.

[Type text] 
Acknowledgments. This work is supported by the Interconnect Focus Center (MARCO program), State of New York, the National Science Foundation (NSF) Integrative Graduate Education and Research Traineeship (IGERT) program, Grant No. 0333314, the NSF Petascale Simulations and Analysis (PetaApps) program, Grant No. 0749140, the Army Research Laboratory under cooperative agreement number W911NF-12-2-0023, and an anonymous gift from Rensselaer. Computing resources of the Center for Computational Innovations (CCI) at Rensselaer, partly funded by the State of New York, have been used for this work.

\section{References}

1 V. Schmidt, J. V. Wittemann, S. Senz, and U. Gosele, Adv. Mater. 21, 2681 (2009).

2 D. D. D. Ma, C. S. Lee, F. C. K. Au, S. Y. Tong, and S. T. Lee, Science 299, 1874 (2003).

3 A. J. Read, R. J. Needs, K. J. Nash, L. T. Canham, P. D. J. Calcott, and A. Qteish, Phys. Rev. Lett. 69, 1232 (1992).

$4 \quad$ X. Zhao, C. M. Wei, L. Yang, and M. Y. Chou, Phys. Rev. Lett. 92, 236805 (2004).

5 J. D. Holmes, K. P. Johnston, R. C. Doty, and B. A. Korgel, Science 287, 1471 (2000).

$6 \quad$ L. T. Canham, Appl. Phys. Lett. 57, 1046 (1990).

$7 \quad$ Y. Cui and C. M. Lieber, Science 291, 851 (2001).

8 D. W. Wang, Q. Wang, A. Javey, R. Tu, H. J. Dai, H. Kim, P. C. McIntyre, T. Krishnamohan, and K. C. Saraswat, Appl. Phys. Lett. 83, 2432 (2003).

9 R. S. Friedman, M. C. McAlpine, D. S. Ricketts, D. Ham, and C. M. Lieber, Nature 434, 1085 (2005).

10 Y. Cui, Z. H. Zhong, D. L. Wang, W. U. Wang, and C. M. Lieber, Nano Lett. 3, 149 (2003).

11 Y. Huang, X. F. Duan, Y. Cui, L. J. Lauhon, K. H. Kim, and C. M. Lieber, Science 294, 1313 (2001).

12 Y. Cui, Q. Q. Wei, H. K. Park, and C. M. Lieber, Science 293, 1289 (2001).

13 A. K. Geim and K. S. Novoselov, Nature Mater. 6, 183 (2007).

14 X. Shen, S. M. Hou, Z. K. Qian, X. Y. Zhao, and J. L. Wu, Physica E 41, 865 (2009).

15 M. Bruno, M. Palummo, A. Marini, R. Del Sole, V. Olevano, A. N. Kholod, and S. Ossicini, Phys. Rev. B 72, 153310 (2005).

16 T. Vo, A. J. Williamson, and G. Galli, Phys. Rev. B 74, 045116 (2006).

17 M. Bruno, M. Palummo, A. Marini, R. Del Sole, and S. Ossicini, Phys. Rev. Lett. 98, 036807 (2007).

18 J.-A. Yan, L. Yang, and M. Y. Chou, Phys. Rev. B 76, 115319 (2007).

19 D. Medaboina, V. Gade, S. K. R. Patil, and S. V. Khare, Phys. Rev. B 76, 205327 (2007).

20 H. Peelaers, B. Partoens, M. Giantomassi, T. Rangel, E. Goossens, G. M. Rignanese, X. Gonze, and F. M. Peeters, Phys. Rev. B 83, 045306 (2011).

21 Y. M. Niquet, A. Lherbier, N. H. Quang, M. V. Fernández-Serra, X. Blase, and C. Delerue, Phys. Rev. B 73, 165319 (2006).

[Type text] 
[Type text]

M. Diarra, C. Delerue, Y.-M. Niquet, and G. Allan, J. Appl. Phys. 103, 073703 (2008). M. Diarra, Y.-M. Niquet, C. Delerue, and G. Allan, Phys. Rev. B 75, 045301 (2007).

C. Li, M. Bescond, and M. Lannoo, Appl. Phys. Lett. 97, 252109 (2010).

J. B. Neaton, M. S. Hybertsen, and S. G. Louie, Phys. Rev. Lett. 97, 216405 (2006).

Y. Li, D. Lu, and G. Galli, J. Chem. Theory Comput. 5, 881 (2009).

N. Kharche and S. K. Nayak, Nano Lett. 11, 5274 (2011).

L. Liang and V. Meunier, Phys. Rev. B 86, 195404 (2012).

X. Jiang, N. Kharche, P. Kohl, T. B. Boykin, G. Klimeck, M. Luisier, P. M. Ajayan, and S. K. Nayak, Appl. Phys. Lett. 103, 133107 (2013).

N. A. Lanzillo, N. Kharche, and S. K. Nayak, Sci. Rep. 4, 3609 (2014).

W. Kohn and L. J. Sham, Phys. Rev. 140, 1133 (1965).

P. Hohenberg and W. Kohn, Phys. Rev. B 136, B864 (1964).

X. Gonze, B. Amadon, P. M. Anglade, J. M. Beuken, F. Bottin, P. Boulanger, F.

Bruneval, D. Caliste, R. Caracas, M. Cote, T. Deutsch, L. Genovese, P. Ghosez, M.

Giantomassi, S. Goedecker, D. R. Hamann, P. Hermet, F. Jollet, G. Jomard, S. Leroux, M. Mancini, S. Mazevet, M. J. T. Oliveira, G. Onida, Y. Pouillon, T. Rangel, G. M. Rignanese, D. Sangalli, R. Shaltaf, M. Torrent, M. J. Verstraete, G. Zerah, and J. W. Zwanziger, Comp. Phys. Comm. 180, 2582 (2009).

X. Gonze, G. M. Rignanese, M. Verstraete, J. M. Beuken, Y. Pouillon, R. Caracas, F. Jollet, M. Torrent, G. Zerah, M. Mikami, P. Ghosez, M. Veithen, J. Y. Raty, V. Olevano, F. Bruneval, L. Reining, R. Godby, G. Onida, D. R. Hamann, and D. C. Allan, Z. Kristallogr. 220, 558 (2005). N. Troullier and J. L. Martins, Phys. Rev. B 43, 1993 (1991).

S. Goedecker, M. Teter, and J. Hutter, Phys. Rev. B 54, 1703 (1996).

S.-M. Choi, S.-H. Jhi, and Y.-W. Son, Phys. Rev. B 81, 081407 (2010).

H.-P. Komsa and A. V. Krasheninnikov, Phys. Rev. B 86, 241201 (R) (2012).

K. Momma and F. Izumi, J. Appl. Crystallogr. 44, 1272 (2011).

J. P. Perdew and M. Levy, Phys. Rev. Lett. 51, 1884 (1983).

L. J. Sham and M. Schluter, Phys. Rev. Lett. 51, 1888 (1983).

L. Hedin, Phys. Rev. 139, A796 (1965).

S. Ismail-Beigi, Phys. Rev. B 73, 233103 (2006).

M. S. Hybertsen and S. G. Louie, Phys. Rev. B 34, 5390 (1986).

C. D. Spataru, S. Ismail-Beigi, L. X. Benedict, and S. G. Louie, Appl. Phys. A-Mater. Sci. Process. 78, 1129 (2004).

Y. Wu, Y. Cui, L. Huynh, C. J. Barrelet, D. C. Bell, and C. M. Lieber, Nano Lett. 4, 433 (2004).

L. J. Lauhon, M. S. Gudiksen, C. L. Wang, and C. M. Lieber, Nature 420, 57 (2002).

A. J. Lu and R. Q. Zhang, Solid State Commun. 145, 275 (2008).

I. D. White, R. W. Godby, M. M. Rieger, and R. J. Needs, Phys. Rev. Lett. 80, 4265 (1998).

A. G. Eguiluz and W. Hanke, Phys. Rev. B 39, 10433 (1989).

F. Huser, T. Olsen and K.S. Thygesen, Phys. Rev. B 87, 235132 (2013) 
(a)

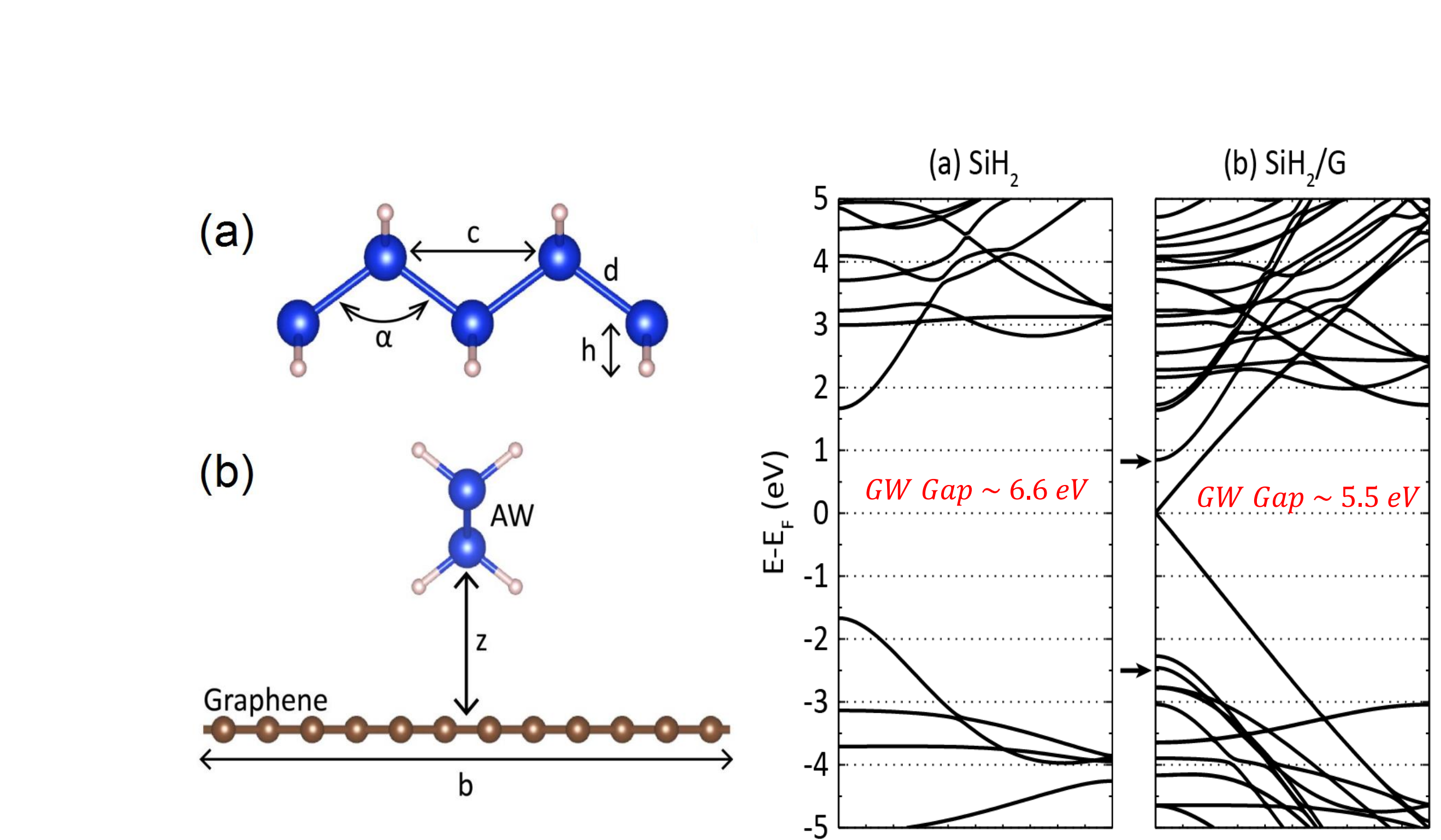

(b)
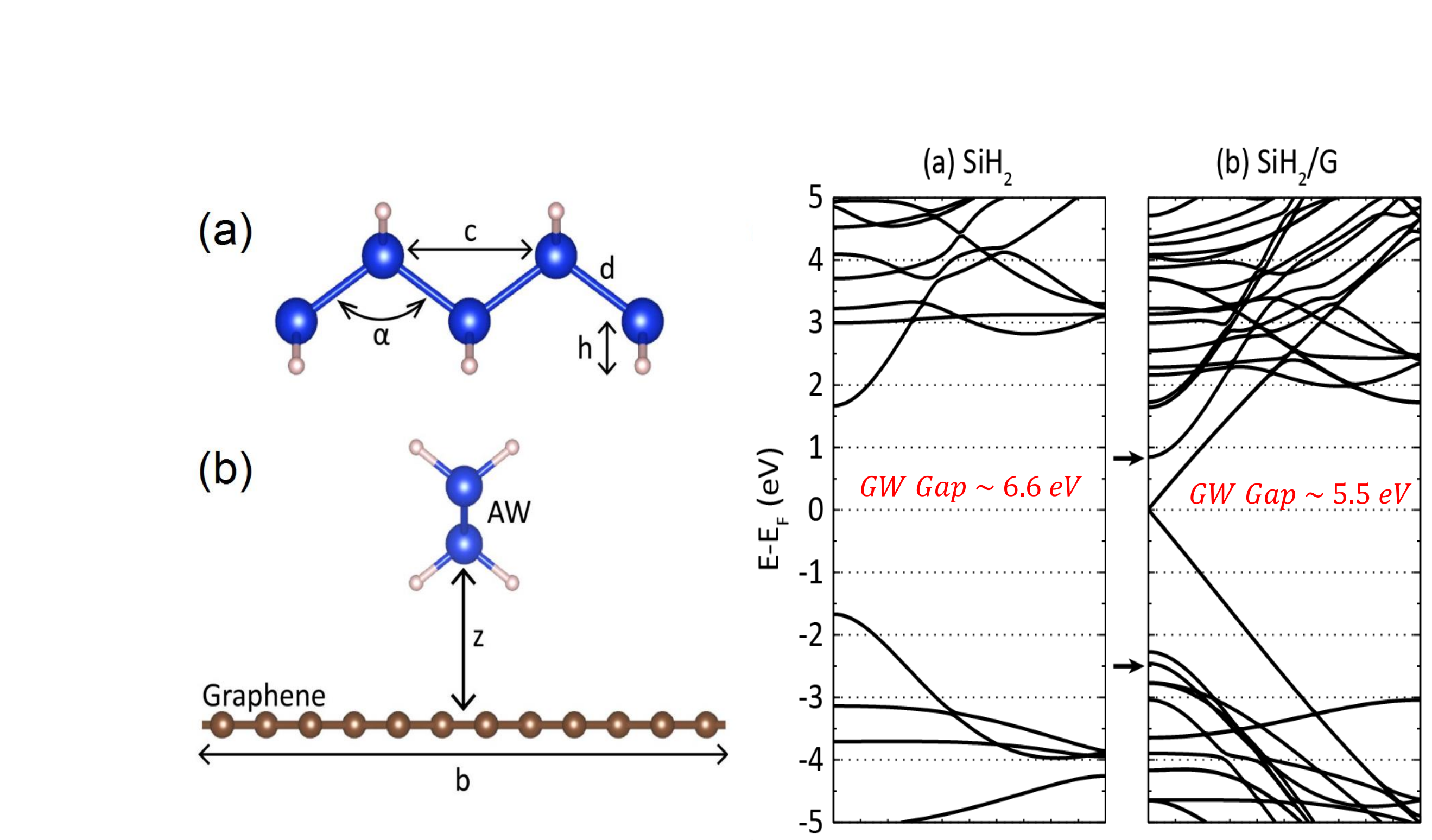

(b) $\mathrm{SiH}_{2} / \mathrm{G}$



